\title{
Constraints on the Angular Distribution of the Zeros of a Polynomial of Low Complexity
}

\author{
Gilbert Stengle \\ Department of Mathematics, Lehigh University, Bethlehem, PA 18015, USA
}

\begin{abstract}
Let $P(z)$ be a polynomial of degree $n$ with complex coefficients. Theorem. There exists a constant $C$ such that if $P$ has at most $k$ terms then the number of zeros of $P$ in any open sector of aperture $\pi / n$ at the origin is no more than $C^{k^{2}}$. The main point of this bound is that it is independent both of $n$ and the coefficients of $P$. The proof is a simple application of Khovanskii's real Bezout theorem to the system of real equations $\operatorname{Re} P=\operatorname{Im} P=0$. We also describe a measure of additive complexity for sets of integers and use it to estimate the angular distribution more finely.
\end{abstract}

\section{Introduction}

A basic method in real geometry is, whenever possible, to transfer results from complex algebraic geometry. This note contains an argument which truly goes in the opposite direction. It gives an application to complex geometry of an essentially real theorem. This is the following extension of Bezout's theorem due to Khovanskii [K1], [K2].

Theorem 1.1 (Khovanskii). Let $\left\langle a_{i}, x\right\rangle, i=1, \ldots, k,\left\langle b_{i}, x\right\rangle, i=1, \ldots, \rho$, be linear forms on $R^{n}$. Let $P_{i}(x, y, u, v), i=1, \ldots, n$, be polynomials of degree $m_{i}$ in the $n+k+2 \rho$ real variables $x_{1}, \ldots, x_{n}, y_{1}, \ldots, y_{k}, u_{1}, \ldots, u_{\rho}, v, \ldots, v_{\rho}$. For each $i$ let $y_{i}=\exp \left\langle a_{i}, x\right\rangle, u_{i}=\cos \left\langle b_{i}, x\right\rangle$, and $v_{i}=\sin \left\langle b_{i}, x\right\rangle$. Then the number of nondegenerate solutions of the system

$$
P_{1}(x, y, u, v)=\cdots=P_{n}(x, y, u, v)=0
$$

satisfying

$$
\left|\left\langle x, b_{i}\right\rangle\right|<\pi / 2, \quad i=1, \ldots, \rho,
$$

does not exceed $m_{1} \cdots m_{n}\left(1+\rho+\sum m_{i}\right)^{(k+\rho)} 2^{\rho+(k+\rho)(k+\rho-1) / 2}$. 
Generically it is a retrograde step to reduce a single complex polynomial equation $P(x+i y)=0$ to the pair of real equations $\operatorname{Re} P=\operatorname{Im} P=0$. For example, since each equation has degree $n$, the classical Bezout estimate for this pair only gives the absurdly weak bound $n^{2}$ for the number of simple zeros of $P$. Nevertheless, this reduction can be useful in the highly nongeneric situation illustrated by the polynomial $z^{n}-a$. The zeros of this polynomial have a discrete uniform angular distribution and no sector of aperture $\pi / n$ at the origin can contain more than one zero. The following is a qualitatively similar result for polynomials with a small number of terms (sometimes called a "fewnomial"). It shows that the angular distribution of the zeros of a polynomial of low complexity (in a sense to be made more precise below) cannot be too sharply concentrated.

Theorem 1.2. There exists a constant $C$ such that if $P$ is any polynomial of degree $n$ with at most $k$ terms, then the number of zeros of $P$ in any open sector of aperture $\pi / n$ at the origin is no more than $C^{k^{2}}$.

Proof. For purposes of the proof let $k$ denote the number of nonconstant terms in $P$. By a change of variable $z \rightarrow z$ exp i $\alpha$ we can assume that the sector in question is $|\arg z|<\pi / 2 n$. If $P$ has multiple zeros, we replace $P$ by $P+\varepsilon$ where the constant $\varepsilon$ is chosen in the following way. $P+\varepsilon$ has multiple zeros for only finitely many values of $\varepsilon$. Excluding such values, by the continuous dependence of the zeros on $\varepsilon$, for $\varepsilon$ sufficiently small, $P+\varepsilon$ has no fewer simple zeros in the open sector than $P$ has zeros counted with multiplicity. We therefore assume that $P$ has only simple zeros. Let $x_{1}=\ln |z|$ and $x_{2}=\arg z,-\pi<x_{2} \leq \pi$. If the monomial $z^{j}(j \neq 0)$ actually appears in $P$ let $y_{j}=\exp j x_{1}, u_{k}=\cos j x_{2}$, and $v_{j}=$ $\sin j x_{2}$. Then, using $z^{j}=y_{j}\left(u_{j}+i v_{j}\right)$, the equations $\operatorname{Re} P=\operatorname{Im} P=0$ take the form $P_{1}(y, u, v)=\dot{P}_{2}(y, u, v)=0$ where the polynomials $P_{j}$ are of total degree 2 . This system has the form required by Khovanskii's theorem with $m_{1}=m_{2}=2$ and $\rho=k$. Hence the number of nondegenerate solutions $\left(x_{1}, x_{2}\right)$ satisfying $\left|j x_{2}\right|<\pi / 2$ is no more than $4(5+k)^{2 k} 2^{2 k^{2}}<C^{k^{2}}$. Since $P$ has degree $n$ the inequality $\left|n x_{2}\right|<$ $\pi / 2$ necessarily occurs among the constraining inequalities and implies the remaining inequalities.

It is evident from the proof that any $C$ larger than 4 will do for sufficiently large $k$. For example, it is easy to check that $C=5.3$ suffices for all $k>10$.

\section{Some Complexity Estimates}

If the set of nonzero exponents actually appearing in $P$ has a simple additive structure, then the estimate of Theorem 1.2 can sometimes be improved.

Proposition 2.1. Suppose that the nonzero exponents actually appearing in $P$ form a subsequence of a $k$-term arithmetic progression $a, a+d, \ldots, a+(k-1) d$. Then the number of zeros of $P$ in any open sector of aperture $\pi / n$ at the origin has an upper bound which is polynomial in $k$. 
Proof. Varying the reformulation of $P=0$ in the proof of Theorem 1.2, again let $x_{1}=|z|, x_{2}=\arg z$, but now introduce $y_{1}=\exp a x_{1}, y_{2}=\exp d x_{1}, u_{1}=\cos a x_{2}$, $u_{2}=\cos d x_{2}, v_{1}=\sin a x_{2}$, and $v_{2}=\sin d x_{2}$. Then $z^{a+j d}=y_{1}\left(y_{2}\right)^{j}\left(u_{1}+i v_{1}\right)\left(u_{2}+i v_{2}\right)^{j}$ and the equations $\operatorname{Re} P=\operatorname{Im} P=0$ have the form $P_{1}(y, u, v)=P_{2}(y, u, v)=0$ where both polynomials have total degree exactly $2 k$ and $y, u$, and $v$ are each twodimensional. The reasoning of Theorem 1.2 then implies that if $\nu=\max (a, d)$, then the number of zeros of $P$ in any open sector of aperture $\pi / \nu$ is not greater than $4 k^{2}(3+4 k)^{4} 2^{8}<C k^{6}$

Example 2.2. The polynomial $(z-1)^{n}-\varepsilon$ has additive complexity 2 but for sufficiently small $\varepsilon$ all of its zeros lie in a sector of any preassigned small aperture.

This example shows that the properties of $P$ which control the angular distribution of its zeros do not depend directly on the additive complexity of $P$ itself $[R]$. Instead it is the additive structure of the set of its exponents. This we proceed to quantify.

Definition 2.3. Let $M(P)$ be the set of nonzero exponents actually appearing in the polynomial $P$.

Given a set $S$ let $|S|$ denote its cardinality.

A coarse, intrinsic measure of the additive complexity of $M(P)$ is given by an integer-valued function $\gamma_{M(P)}$ derived from the following notion of the additive complexity function of a set.

Definition 2.4. Let $M, G$ be subsets of a commutative semigroup $\mathfrak{N}$. Let the diameter of $M$ with respect to $G, d(M, G)$, be $\infty$ if there is no $k$ for which $M \subset G \cup(G+G) \cup \cdots \cup(G+G+\cdots+G)(k$ summands $)$ and otherwise the minimum $k$ for which such an inclusion holds. Let the additive complexity function of $M$ be

$$
\gamma_{M}(s)=\min \{d(M, G)|| G \mid=s\} .
$$

The function $\gamma_{M}$ measures the disposition of $M$ to be generated additively by sets of smaller cardinality. It is obvious that $\gamma_{M}(s)$ is a nonincreasing function of $s$ and that $\gamma_{M}(s)=1$ for $s \geq|M|$. From this point on our attention is restricted to the special case $G=\mathbb{N}$.

Example 2.5. Let $M=\{1,2,4,8,16,31\}$. Then it is not hard to check that $\gamma(1)=$ $31, \gamma(2)=6, \gamma(3)=3, \gamma(4)=2, \gamma(5)=2$, and $\gamma(6)=1$.

In the following propositions we find upper estimates for $\gamma_{M}$ by considering special sets of generators. The first is an explicit, noncombinatorial bound.

Proposition 2.6. List the elements of $M$ as an increasing sequence $m_{1}, m_{2}, \ldots, m_{k}$. Let $d_{j}=\operatorname{gcd}\left(m_{1}, \ldots, m_{j}\right)$. Then

$$
\gamma_{M}(j) \leq \min _{1 \leq i \leq j} i\left(m_{k-j+i} / d_{k-j+i}\right)^{1 / i}
$$


Proof. Induction on $k=|M|$. For $k=1$ the assertion is trivial. For general $k$ and $j=1$ the best generating set is $\left\{d_{k}\right\}$ and the hardest element to generate is $m_{k}$ which requires $m_{k} / d_{k}$ summands. For $j>1$ consider the $j$-element generating set $\left\{d_{k}, d_{k} p_{1}, \ldots, d_{k} p_{j-1}\right\}$ where $p_{i}$ is the smallest integer greater than $\left(m_{k} / d_{k}\right)^{i / j}$. We show that each $m_{i}$ is the sum of no more than $j\left(m_{k} / d_{k}\right)^{1 / j}$ members of this set. To this end let $i \in\{1,2, \ldots, k\}$ be given. By the division algorithm,

$$
m_{i} / d_{k}=q_{j-1} p_{j-1}+r_{j-1}
$$

where

$$
q_{j-1} \leq\left(m_{i} / d_{k}\right)\left(m_{k} / d_{k}\right)^{-(j-1) / j} \leq\left(m_{k} / d_{k}\right)^{1 / j}
$$

and

$$
r_{j-1} \leq p_{j-1}-1 \leq\left(m_{k} / d_{k}\right)^{(j-1) / j}
$$

Applying the division algorithm to $r_{j-1}$ we have

$$
r_{j-1}=q_{j-2} p_{j-2}+r_{j-2}
$$

where

$$
q_{j-2} \leq r_{j-1}\left(m_{k} / d_{k}\right)^{-(j-2) / j} \leq\left(m_{k} / d_{k}\right)^{(j-1) / j}\left(m_{k} / d_{k}\right)^{-(j-2) / j}=\left(m_{k} / d_{k}\right)^{1 / j}
$$

and

$$
r_{j-2} \leq p_{j-2}-1 \leq\left(m_{k} / d_{k}\right)^{(j-2) / j}
$$

Repeated application yields

$$
\begin{aligned}
r_{j-2} & =q_{j-3} p_{j-3}+r_{j-3}, \\
\vdots & \\
r_{2} & =q_{1} p_{1}+r_{1},
\end{aligned}
$$

where each $q_{t} \leq\left(m_{k} / d_{k}\right)^{1 / j}$ and $r_{1} \leq p_{1}-1 \leq\left(m_{k} / d_{k}\right)^{1 / j}$. Combining these equations we have

$$
m_{i} / d_{k}=\sum_{t=1}^{j-1} q_{t} p_{t}+r_{1}
$$

where the $q_{t}$ and $r_{1}$ have the common bound $\left(m_{k} / d_{k}\right)^{1 / j}$. Thus

$$
m_{i}=\sum_{t=1}^{j-1} q_{i}\left(d_{k} p_{i}\right)+r_{1} d_{k}
$$

which is a sum of $\sum_{t=1}^{j-1} q_{t}+r_{1}$ generators. Since this sum is bounded by $j\left(m_{j} / d_{k}\right)^{1 / j}$ we have established

$$
\gamma_{M}(j) \leq j\left(m_{k} / d_{k}\right)^{1 / j}
$$


We now consider generation of $M$ by special $j$-element sets containing the largest element $m_{k}$. Let $M^{\prime}=\left\{m_{1}, \ldots, m_{k-1}\right\}$. It is obvious that $\gamma_{M}(j) \leq \gamma_{M^{\prime}}(j-1)$. By induction hypothesis

$$
\gamma_{M} \cdot(j-1) \leq \min _{1 \leq i \leq j-1} i\left(m_{k-1-(j-1)+i} / d_{k-1-(j-1)+1}\right)^{1 / i}
$$

Hence

$$
\gamma_{M}(j) \leq \min _{1 \leq i \leq j-1} i\left(m_{k-j+i} / d_{k-j+i}\right)^{1 / i}
$$

Estimates $(*)$ and $(* *)$ together imply the asserted conclusion.

The following shows that if the elements of $M$ are very widely spaced, then $M$ can have only trivial additive structure. In such a case the most effective use of generators is simply to account for the largest possible elements of $M$.

Corollary 2.7. Let the elements of $M$ form an increasing sequence $m_{1}, \ldots, m_{k}$. Let $d_{j}=\operatorname{gcd}\left(m_{1}, \ldots, m_{j}\right)$. If $m_{i+1}>m_{i}^{2} / d_{i}$ for each $i$, then the $j$-element set

$$
M_{j}=\left\{d_{k-\jmath+1}, m_{k-j+2}, \ldots, m_{k}\right\}
$$

generates $M$ most efficiently in the sense that $\gamma_{M}(j)=d\left(M, M_{j}\right)=m_{k-j+1} / d_{k-j+1}$.

Proof. The asserted value for $\gamma_{M}(j)$ occurs as the value of the bound of Proposition 2.6 when the indicated minimum in this bound is attained for $i=1$. This is the case provided for each $i$ and $j$ :

$$
j\left(m_{i+1} / d_{i+1}\right)^{1 / j} \geq(j-1)\left(m_{i} / d_{i}\right)^{1 /(j-1)}
$$

or equivalently

$$
m_{i+1} \geq(1-1 / j)^{j}\left(m_{i} / d_{i}\right)^{j /(j-1)} d_{i+1} .
$$

Since $(1-1 / j)^{j}<1$ and $j /(j-1) \leq 2$ and $d_{i+1} \leq d_{i}$, these inequalities follow from our hypotheses. Thus

$$
\gamma_{M}(j) \leq m_{k-j+1} / d_{k-j+1}
$$

and this bound is attained for the generating set $\boldsymbol{M}_{j}$.

We now show by induction on $k=|M|$ that the bound of $(* * *)$ is also a lower bound. For $k=1$ the conclusion is trivial. For $k>1$ let $G_{j}$ be any set such that $\gamma_{M}(j)=d\left(M, G_{j}\right)$ and let $g^{\prime}$ be its largest element. For $j=1$ equality in (***) is trivial so assume $j>1$. Then the number of summands in any representation of $m_{k}$ cannot be less than $m_{k} / g^{\prime}$. Hence $\gamma_{M}(j) \geq m_{k} / g^{\prime}$. Hence, by $(* * *), m_{k} / g^{\prime} \leq$ $m_{k-j+1} / d_{k-j+1} \leq m_{k-1} / d_{k-1}$ or $g^{\prime} \geq m_{k} d_{k-1} / m_{k-1}$. But in any representation of an element of $M$ other than $m_{k}, g^{\prime}$ can appear no more than $m_{k-1} / g^{\prime}$ times as a summand. Since $m_{k-1} / g^{\prime} \leq m_{k-1}^{2} / d_{k-1} m_{k}<1$, in fact, $g^{\prime}$ cannot occur as a summand in a representation of any element other than $m_{k}$. Hence without imparing the optimality of $G^{\prime}$ we can replace $g^{\prime}$ by $m_{k}$ and also $\gamma_{M}(j)=\gamma_{M \backslash\left\{m_{k}\right\}}(j-1)$. By induction hypothesis this latter function has the asserted form. 


\section{A Combinatorial Refinement}

In terms of the function $\gamma_{M(P)}$ we can formulate a generalization of Theorem 1.2 which also covers Proposition 2.1.

Theorem 3.1. There exists a constant $C$ such that if $P$ is a complex polynomial of degree $n, M$ is the set of nonzero exponents actually appearing in $P, \gamma_{M}$ is the additive complexity function of $M$, and

$$
\kappa(P)=\min _{j>0}\left[(j+1) \log _{2} \gamma_{M}(j)+j^{2}\right]
$$

then any open sector of aperture $\pi / n$ at the origin contains no more than $C^{\kappa(P)}$ zeros of $P$.

Proof. Let $h(\gamma, k)=4 \gamma^{2}(1+2 \gamma+2 k)^{2 k} 2^{2 k^{2}}$. Then elementary estimation shows

$$
\log _{2} h<A\left[(k+1) \log _{2} \gamma+k^{2}\right]
$$

for a suitable constant $A$. For each $k$ between 1 and $|M|$ the set $M$ is generated additively by a $k$-element set $M^{\prime}$ where each element of $M$ is a sum of no more than $\gamma_{M}(k)$ elements. Again we introduce the variables $x_{1}=\ln |z|, x_{2}=\arg z$, and, for each $m$ in $M^{\prime}, y_{m}=\exp m x_{1}, u_{m}=\cos m x_{2}, v_{m}=\sin m x_{2}$. The equations $\operatorname{Re} P=\operatorname{Im} P=0$ then assume the form $P_{1}(y, u, v)=P_{2}(y, u, v)=0$ where the polynomials $P_{j}$ have degree $2 \gamma_{M}(k)$ and the variables $y, u$, and $v$ are each $k$ dimensional. Reprising the reasoning of Theorem 1.2 we find that any open sector of aperture $\pi / n$ contains no more than $h\left(\gamma_{M}(k), k\right)$ zeros of $P$. Using the above estimate for $\log _{2} h$ and choosing the $k$ which optimizes this estimate we obtain the asserted conclusion.

This contains Theorem 1.2 since $\kappa(P) \leq(|M|+1) \log _{2}\left(\gamma_{M}(|M|)+|M|^{2}\right)=|M|^{2}$. It also implies Proposition 2.1 since if $M \subset\{a, a+d, \ldots, a+[k-1] d)$, then $\beta(M,\{a, d\})=k$ which implies $\gamma_{M}(2) \leq k$. This in turn implies $\kappa(P) \leq 3 \log _{2} k+4$ which yields a polynomial estimate for $C^{\kappa(P)}$. We remark that $\{a, d\}$ is not in general a most efficient two-element generating set for $M$.

In conclusion we raise the question of suitable algorithms for computing either exact values or upper bounds for $\gamma_{M}$. Here, with the help of Murray Schechter, we have made small experiments with machine computation of $\gamma_{M}$, pruning the testing of generating sets with the estimate of Proposition 2.6 and using backtracking to find optimal representations of individual integers. In reasonable computation times we have only found examples which lie slightly beyond those that can be treated by hand calculation. For example, if

$$
M=\{1,155,156,331,10033\}
$$


then $\gamma(1)=1033, \gamma(2)=16, \gamma(3)=6, \gamma(4)=2$, and $\gamma(5)=1$. This is to be expected since our problem consists essentially of an exhaustive search of a family of subset sum problems which are known to be NP-complete. Still it would be interesting to find a heuristic which is responsive to the special features of the present application.

\section{References}

[K1] A. Khovanskii, On a class of systems of transcendental equations, Soviet Math. Dokl. 22 (3) (1980), 762-765.

[K2] A. Khovanskii, Sur les racines complexes de systems d'équations algébriques, C. R. Acad. Sci. Paris 292 (1981), 937-940.

[R] J. J. Risler, Additive complexity and zeros of real polynomials, SIAM J. Comput. 14 (1) (1985), $178-183$.

Received June 23, 1988, and in revised form December 8, 1988. 